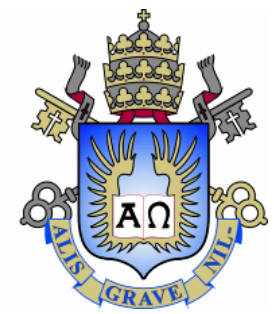

Vinicius Guimarães Rodrigues

A interação entre informações linguística e visual na compreensão da linguagem

Dissertação de Mestrado

Dissertação apresentada como requisito parcial para obtenção do grau de Mestre pelo Programa de Pósgraduação Estudos da Linguagem do Departamento de Letras da PUC-Rio.

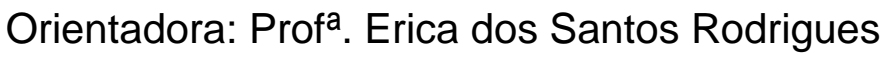




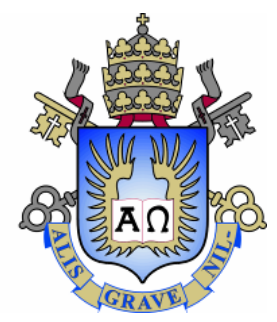

\title{
Vinicius Guimarães Rodrigues
}

\section{A interação entre informações linguística e visual na compreensão da linguagem}

Dissertação apresentada como requisito parcial para obtenção do grau de Mestre pelo Programa de Pósgraduação Estudos da Linguagem do Departamento de Letras da PUC-Rio. Aprovada pela Comissão Examinadora abaixo assinada.

\author{
Profa. Erica dos Santos Rodrigues \\ Orientadora
}

Prof ${ }^{a}$. Mercedes Marcilese

Departamento de Letras - Universidade Federal de Juiz de Fora

Prof. Eduardo Kenedy Nunes Areas Departamento de Letras - Universidade Federal Fluminense

Profa. Denise Berruezo Portinari Coordenadora Setorial do Centro de Teologia e Ciências Humanas - PUC-Rio 
Todos os direitos reservados. É proibida a reprodução total ou parcial do trabalho sem a autorização da universidade, do autor e da orientadora.

\section{Vinicius Guimarães Rodrigues}

Graduou-se em Comunicação Social na UFRJ (Universidade Federal do Rio de Janeiro) em 2003. É professor de inglês no Rio de Janeiro, com experiência, particularmente, no ensino de inglês como língua estrangeira para adultos.

Ficha Catalográfica

Rodrigues, Vinicius Guimarães

A interação entre informações linguística e visual na compreensão da linguagem / Vinicius Guimarães Rodrigues; orientadora: Erica dos Santos Rodrigues. 2013.

133 f. : il. (color.) ; 29,7 cm

Dissertação (mestrado) - Pontifícia Universidade Católica do Rio de Janeiro, Departamento de Letras, 2013.

Inclui referências bibliográficas.

1. Letras - Teses. 2. Interface linguagem-visão. 3. Processamento de sentenças. 4. Compreensão da linguagem. 5. Psicolinguística. I. Rodrigues, Erica dos Santos. II. Pontifícia Universidade Católica do Rio de Janeiro. Departamento de Letras. III. Título. 
A meus pais, Antonio José e Maria Helena, e a minha irmã, Fernanda, pelo constante incentivo. 


\section{Agradecimentos}

À Professora Erica dos Santos Rodrigues pelo tempo e energia dispensados na produção desta dissertação.

À PUC-Rio, pelos auxílios concedidos, sem os quais este trabalho não poderia ser realizado.

A minha prima Camylle Guimarães Scheliga, pelo apoio.

A Adriano Koshiyama, pela ajuda na análise estatística.

Aos professores que participaram da Comissão examinadora.

À todos os professores e funcionários do Departamento de Letras, em especial à Chiquinha, pelos ensinamentos e pela ajuda.

A todos os alunos da PUC-Rio que participaram dos experimentos. 


\section{Resumo}

Rodrigues, Vinicius Guimarães; Rodrigues, Erica dos Santos. A interação entre informações linguística e visual na compreensão da linguagem. Rio de Janeiro, 2013. 133p. Dissertação de Mestrado - Departamento de Letras, Pontifícia Universidade Católica do Rio de Janeiro.

Esta dissertação tem por objetivo analisar como ocorre a interação entre informações de ordens linguística e visual em experimentos de compreensão de linguagem. Sentenças do português nas vozes ativa e passiva e com verbos de perspectiva (perseguir/fugir) foram analisadas a partir da técnica de comparação sentença-imagem, com manipulação de posição ocupada na imagem pelo personagem correspondente ao sujeito da sentença (esquerda vs. direita) e também do papel temático (agente/fonte $v s$. paciente/alvo) de um dos personagens sobre o qual recaía um recurso de manipulação de atenção visual. Do ponto de vista teórico, problematizou-se a interação entre conteúdos proposicionais oriundos do processamento visual e linguístico com base em uma articulação entre a faculdade da linguagem no sentido amplo (Hauser, Chomsky \& Fitch, 2002) e a teoria da modularidade da mente (Fodor, 1983). Os resultados de dois experimentos realizados com falantes de português sugerem que, no mapeamento sentença-imagem, a posição do personagem correspondente ao sujeito não parece ser um fator relevante, a não ser em estruturas mais complexas, como no caso de sentenças envolvendo verbos de perspectiva em que o papel temático do sujeito não é prototípico. Em relação a papel temático, o fato de o foco atencional numa imagem estar sobre um elemento que corresponde a um sujeito com papel prototípico de agente parece facilitar o processamento. Quanto aos tipos de estrutura, como já verificado na literatura psicolinguística, estruturas ativas parecem ser menos complexas do que passivas e facilitam o mapeamento visual. Quanto à expressão de perspectiva, verbos de perspectiva 
que empreendem um sujeito paciente/alvo parecem demandar maior custo de processamento, possivelmente em função de questões de acesso e representação lexical associadas a esses verbos.

\section{Palavras-chave}

Interface linguagem-visão; processamento de sentenças; compreensão da linguagem; Psicolinguística 


\section{Abstract}

Rodrigues, Vinicius Guimarães; Rodrigues, Erica dos Santos (Advisor). The interaction between linguistic and visual information in language comprehension. Rio de Janeiro, 2013. 133p. MSc. Dissertation - Departamento de Letras, Pontifícia Universidade Católica do Rio de Janeiro.

This dissertation aims to analyze how the interface between linguistic and visual information occurs based on language comprehension experiments. Active and passive voice sentences, as well as perspective predicates were analyzed using a sentence-image verification technique. The position of the characters representing the subject were manipulated (left-right orientation), and so were their theme roles (agent/source vs. patient/target) by means of attention manipulation. The propositional theory, a relation between the faculty of language in the broad sense (Hauser, Chomsky \& Fitch, 2002) and the modularity of mind theory (Fodor, 1983) were presented so as to explain how the interface between linguistic and visual information occurs. Our experiments were carried out with Portuguese speakers, and results indicate that during sentence-image mapping, left-right orientation does not seem to be a relevant factor, except for more complex structures, such as perspective predicates where the subject's theme role is not prototypical. As for theme roles, the attention manipulation on the subject character seems to facilitate language processing. As for sentence types, active voice sentences seem to be processed more easily than passive ones. Perspective predicates whose subjects take a "passive/target" role seem to demand higher processing costs, possibly because of lexical access and representation.

\section{Keywords}

Language-vision interface; sentence processing; language comprehension; Psycholinguistics 


\section{Sumário}

1. Apresentação e justificativa 16

1.1. Objetivos 18

1.2. Hipótese de trabalho 18

1.3. Possíveis contribuições da pesquisa 19

1.4. Organização do trabalho 19

2. Percepção visual e processamento da imagem 21

2.1. Percepção visual: aspectos biológicos 21

2.2. A caracterização da visão segundo Marr (1982) 25

2.3. Percepção e representação de estímulos visuais 28

2.3.1. A teoria da dupla codificação 28

2.3.2. A teoria proposicional 32

2.3.3. Atenção visual e linguagem 36

3. Modularidade da mente e a interface linguagem-visão 40

3.1. A teoria da modularidade da mente 40

3.2. A arquitetura da interface linguístico-espacial proposta 46 por Jackendoff

3.3. A faculdade da linguagem nos sentidos amplo e estrito 49

3.4. A integração linguagem-visão: uma proposta alternativa 52 à de módulos híbridos de Jackendoff

4. Interação linguagem-visão: estudos psicolinguísticos 55

4.1. Apresentação 56

4.2. Estudos relacionando compreensão de linguagem e in- 56 formação visual

4.2.1. Processos preditivos e o paradigma do mundo visual 56

4.2.2. Teoria da Inter-Relação Coordenada (Coordinated Inter- 61 play Account - CIA) da compreensão 
4.3. Estudos relacionando produção de linguagem e infor- 63 mação visual

4.3.1. O estudo de Griffin \& Bock (2000): "What the eyes say 64 about speaking"

4.3.2. O estudo de Gleitman et al. (2007): "On the give and take 66 between event apprehension and utterance formulation"

4.3.3. A relação entre a saliência de elementos no display e a 72 escolha lexical na produção

4.4. Uma proposta de integração entre informação linguís- 75 tica e visual (Clark \& Chase, 1972)

5. Metodologia 77

5.1. A técnica de comparação sentença-figura e o recurso 77 de manipulação de atenção

5.2. Experimentos 79

5.2.1. Experimento 1 - fotografias e sentenças 81

5.2.2. Experimento 2 - desenhos e sentenças 100

6. Considerações finais 109

$\begin{array}{ll}\text { 7. Referências bibliográficas } & 117\end{array}$

8. Anexo 123

8.1. Anexol 123

8.2. Anexo II 130 


\section{Lista de figuras}

Figura 1 - Esquema com algumas áreas responsáveis 23 pelo processamento visual no córtex cerebral (Fulton, 2000).

Figura 2 - Esquema de representação de estímulos ver- 30 bais e visuais segundo a teoria da dupla codificação (adaptado de Clark \& Paivio, 1991, p. 152)

Figura 3 - Concepção de representação mental imagética 35 criticada por Pylyshyn (2003)

Figura 4 - Sugestão de representação mental (conteúdo 35 proposicional) que seria armazenada na memória de acordo com Pylyshyn (2003)

Figura 5 - Experimento com pista de atenção central de 37 Posner (1980, p. 6)

Figura 6 - Sequência de animação "The Fish Film" com 39 manipulação de atenção (seta) utilizada por Tomlin (1997).

Figura 7 - llusão de Müller-Lyre

Figura 8 - Esquema grosseiro da relação entre linguagem 47 e visão (Jackendoff, 1996)

Figura 9 - Proposta alternativa de Rodrigues et al. (2013) 53 de interface linguagem-visão

Figura 10 - Imagem exibida aos participantes quando ou- 57 viam "The boy will eat" ou "The boy will move" no experimento de Altmann \& Kamide (1999)

Figura 11 - Imagem utilizada no experimento de Knoerfele 62 \& Crocker (2006)

Figura 12 - Imagem utilizada no experimento de Griffin \& 65 Bock (2000)

Figura 13 - Imagens utilizadas no experimento de Gleit- 67 man et al. (2007) 
Figura 14 - Sequência de exposição da manipulação de 68 atenção e cena com a instrução para descrição verbal utilizada no experimento de Gleitman et al. (2007)

Figura 15 - Sequência de estímulos (ordem sentença/fo- 86 tografia)

Figura 16 - Sequência de estímulos (ordem fotografia/sen- 86 tença)

Figura 17 - Sequência de estímulos utilizada no experi- 104 mento 2 


\section{Lista de gráficos}

Gráfico 5.2.1.1 - Experimento 1 - ordem sentença/fotografia: mé- 88 dias de tempo de resposta (ms) para as variáveis tipo de sentença e posição do elemento correspondente ao sujeito da sentença Gráfico 5.2.1.2 - Experimento 1 - ordem sentença/fotografia: mé- 89 dias de tempo de resposta (ms) para a variável perspectiva expressa pelo verbo

Gráfico 5.2.1.3 - Experimento 1 - ordem sentença/fotografia: mé- 90 dias de tempo de resposta (ms) para as variáveis perspectiva expressa pelo verbo e posição do sujeito

Gráfico 5.2.1.4 - Experimento 1 - ordem fotografia/sentença: mé- 91 dias de tempo de resposta (ms) - sentenças ativas e passivas Gráfico 5.2.1.5 - Experimento 1 - ordem fotografia/sentença: mé- 92 dias de tempo de reposta $(\mathrm{ms})$ para as variáveis tipo de sentença e posição do sujeito

Gráfico 5.2.1.6 - Experimento 1 - ordem fotografia/sentença: mé- 93 dias de tempo de resposta (ms) para a variável expressão de perspectiva

Gráfico 5.2.1.7 - Experimento 1 - ordem fotografia/sentença: mé- 94 dias de tempo de resposta (ms) para a variável posição do sujeito Gráfico 5.2.1.8 - Experimento 1- ordem fotografia/sentença: mé- 94 dias de tempo de resposta (ms) para as variáveis perspectiva expressa pelo verbo e posição do sujeito

Gráfico 5.2.1.9 - Experimento 1: médias de tempo de resposta 96 (ms) considerando os dois grupos de participantes em conjunto sentenças ativas e passivas

Gráfico 5.2.1.10 - Experimento 1: médias de tempo de resposta 97 (ms) para as variáveis tipo de sentença e posição do sujeito considerando os dois grupos de participantes em conjunto 
Gráfico 5.2.1.11 - Experimento 1: médias de tempo de resposta 97 (ms) por expressão de perspectiva pelo verbo considerando os dois grupos de participantes em conjunto

Gráfico 5.2.1.12 - Experimento 1: médias de tempo de resposta 98 (ms) considerando as variáveis expressão de perspectiva do verbo e posição do sujeito

Gráfico 5.2.1.13 - Experimento 1: médias de tempo de resposta 99 (ms) considerando as variáveis ordem dos estímulos e posição do sujeito

Gráfico 5.2.2.1 - Experimento 2: médias de tempo de resposta das 105 sentenças ativas e passivas das listas 1 e 2 em conjunto Gráfico 5.2.2.2 - Experimento 2: médias de tempo de resposta 106 (ms) para as variáveis tipo de sentença e foco atencional Gráfico 5.2.2.3 - Experimento 2: médias de tempo de resposta 106 (ms) para a variável expressão de perspectiva pelo verbo Gráfico 5.2.2.4 - Experimento 2: médias de tempo de resposta 107 (ms) para as variáveis tipo de perspectiva expressa pelo verbo e foco atencional 


\section{Lista de tabelas}

Tabela 1 - Lista de condições experimentais e exemplos de sen- 83 tenças e fotografias utilizadas no experimento 1

Tabela 2 - Exemplos de sentenças experimentais e figuras utiliza- 102 das no experimento 2

Tabela 3 - Médias gerais de tempo de resposta (ms) para as vari- 108 áveis tipo de sentença e expressão de perspectiva pelo verbo 